\title{
Multiple primary malignancies: A case report of Gastrointestinal stromal tumour (GIST) and Ovarian dermoid cyst
}

\author{
Authors: Manuca Maria Sorana ${ }^{1}$, Prof. MD PhD Eugen Târcoveanu ${ }^{2}$ (mentor) \\ 1 "Grigore T. Popa" University of Medicine and Pharmacy, lasi, Romania \\ ${ }^{2}$ Department of General Surgery, "Grigore T. Popa" University of Medicine and Pharmacy, Saint \\ Spiridon County Hospital, Iasi, Romania
}

DOI: https://doi.org/10.26800/LV-142-supp5-23

\section{Background:}

Multiple primary tumors are defined as more than one tumor in different sites and/or are of a different histology in the same patient. Gastrointestinal stromal tumors (GISTs) are the most common mesenchymal neoplasms of the gastrointestinal tract. Dermoid cysts are benign germ cell tumours which make up to $10-25 \%$ of all ovarian tumours and are usually asymptomatic.

\section{Case presentation:}

We report the case of a 60 years old female with gastrointestinal stromal tumor and left ovarian dermoid cyst who was addressed with tachycardia, pallor, fatigue, faintness and melena. Medical history included bloody stool for 2 months. The endoscopic evaluation revealed a protrusion into the gastric lumen on the posterior wall. Clinical examination revealed no palpable abdominal mass, a soft abdomen with epigastric tenderness. Upper GI series showed a $10 / 5 \mathrm{~cm}$ vegetant mass with a $2 \mathrm{~cm}$ ulcerated and hemorrhagic area on the posterior aspect of the stomach. Abdominal radiography showed calcifications in the left paravesical space. The abdominal ultrasound confirmed the presence of a hypoechogenic mass on the gastric wall and a mixed echo-pattern on the left ovary. Exploratory laparoscopy revealed a $7 \mathrm{~cm}$ cystic mass with smooth surface on the left ovary. Laparoscopic adnexectomy was performed and the cyst removed. For the gastric tumor the patient underwent laparoscopic wedge resection using three ports and linear stapling. Extraction was done by Endobag through umbilical port site. Immunohistochemical staining was positive for Ki167, CD34 and negative for S100 protein.

\section{Conclusion:}

Laparoscopic treatment has proven that it is well associated with low morbidity, mortality, fast recovery, less pain and good oncologic outcomes. For asymptomatic ovarian tumors early recognition and intervention are necessary to avoid potential complications.

Keywords: gastrointestinal stromal tumor, GIST, laparoscopy, multiple primary tumors, ovarian dermoid cyst 